\title{
Effect of Temperature and Humidity on Degradation of Single-Crystal Silicon Microbeam in MEMS Resonator
}

\author{
Taeko Ando, ${ }^{*}$ Mitsuhiro Shikida, ${ }^{1}$ and Kazuo Sato ${ }^{2}$ \\ Department of Mechanical Engineering, Ritsumeikan University, \\ 1-1-1 Noji-Higashi, Kusatsu, Shiga 525-8577, Japan \\ ${ }^{1}$ Graduate School of Information Sciences, Hiroshima City University, \\ 3-4-1 Ozuka-Higashi, Asa-Minami-Ku, Hiroshima 731-3194, Japan \\ ${ }^{2}$ Department of Mechanical Engineering, Aichi Institute of Technology, \\ 1247 Yachigusa, Yakusa-cho, Toyota, Aichi 470-0392, Japan
}

(Received October 6, 2015; accepted December 28, 2015)

Keywords: single-crystal silicon, fatigue strength, temperature, humidity, fracture surface

The fatigue property of single-crystal silicon was studied as a function of environmental conditions, both temperature and humidity, using a fan-shaped resonator. The fatigue tests were conducted in a custom-made measurement setup consisting of an environmental chamber, a heating system, a moisture generator unit, an electrical control system, and an observation system. As a result, it was found that the fatigue strength was markedly decreased under a highly humid condition, but the decrease in fatigue strength due to high humidity was suppressed at a high temperature. Scanning electron microscopy (SEM) observation of the fracture surface of a specimen revealed that the morphologies of the fracture surface depended on the applied stress without both temperature and humidity effects.

\section{Introduction}

Understanding the mechanical degradation of a structural material in a MEMS device is crucial for reliable use and commercial success. The fatigue fracture of silicon, which is the most common as a MEMS structural material, occurs depending on the magnitude of applied stress and environmental conditions such as temperature, humidity, and ambient gasses. It is important to clarify the relationship between the fatigue characteristics of silicon and environmental conditions.

The susceptibility of a silicon microstructure to environmental conditions is well known. Mechanical properties such as density and elastic modulus vary with temperature, as with other materials. It is reported that plastic deformation or ductile behavior can be observed in singlecrystal silicon at low temperature close to room temperature when the representative length of the structure becomes smaller down to the microscale ${ }^{(1,2)}$ or nanoscale. ${ }^{(3)}$ The dislocation generation and migration caused in micro- or nanoscale silicon are more sensitive to environmental temperature. ${ }^{(4,5)}$ As long as silicon is protected from the surrounding environment by sealing in a high-vacuum region, silicon shows long-term stability under resonant condition. ${ }^{(6,7)}$ Fatigue fracture under cyclic loading is accelerated by the existence of moisture for both single-crystal ${ }^{(8-12)}$ and polycrystal silicon. ${ }^{(13-17)}$ Fatigue damage in polysilicon is postulated to be caused by the existence

${ }^{\bar{*}}$ Corresponding author: e-mail: tando@fc.ritsumei.ac.jp 
of a $\mathrm{SiO}_{2}$ layer at a stress-concentrated area ${ }^{(13)}$ or the existence of a damage layer on or in a silicon. ${ }^{(14)}$ The effect of an absorbed water layer on the silicon surface cannot be ignored by the fact that moisture accelerates fatigue damage. Major factors influencing the strength of single-crystal silicon are temperature and humidity under long cyclic conditions. Especially in the microscale, a lower temperature near room temperature is significant to the mechanical behavior, such as deflection and fracture. However, the effect of the combination of temperature and humidity on the fatigue properties has been rarely studied. ${ }^{(8,15)}$

The purpose of this research was to study the effect of both temperature and humidity on the fatigue property of single-crystal silicon in the microscale. By using a micromachined silicon resonator with comb electrodes, tension-compression high-cycle fatigue tests can be performed in an environmental chamber and fatigue failures were detected as a function of environmental conditions.

\section{Materials and Methods}

\subsection{Fatigue testing device structure}

Figure 1 shows the structure of a fatigue testing device used in this study. A movable part with a fan-type configuration is supported by a beam-type specimen, which has a notch on a single side for producing extremely high stress concentration at a local area. Electrostatic force is generated by comb electrodes arranged along both the straight lines of a fan-shaped movable part. Each comb electrode is capable of generating an electrostatic force of $2.5 \mu \mathrm{N}$ at an applied voltage of $100 \mathrm{~V}$. Electrostatic actuators are needed to cause in-plane motion for tilting the movable part and thus stress concentration is generated at the notch tip.

Boron-doped CZ-P-type single-crystal silicon with low resistivity below $0.02 \Omega \mathrm{cm}$ is the testing material in this study. The test specimens were fabricated from silicon-on-insulator (SOI) wafer using conventional micromachining technology. First, metal electrodes were patterned on the top surface of an SOI wafer by a lift-off process of Au. Second, a 10- $\mu$ m-thick top silicon layer was etched by deep reactive ion etching (DRIE). The DRIE process consists of a sequence of $3 \mathrm{~s}$ of

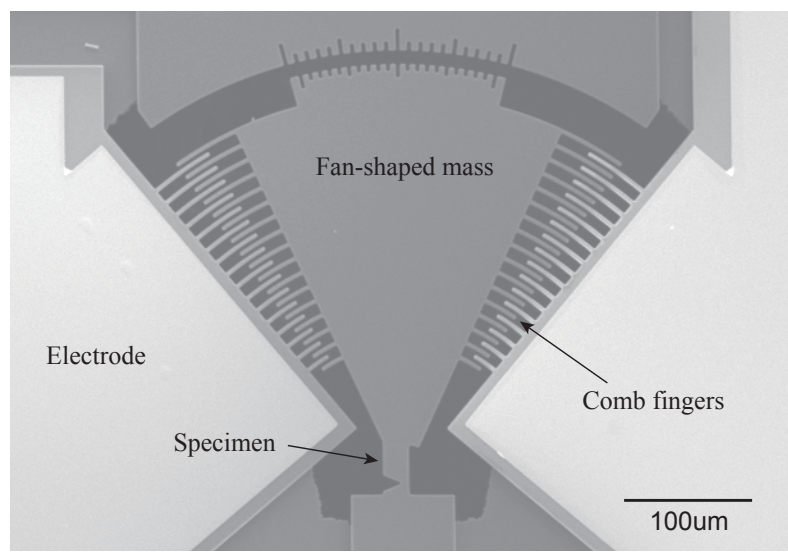

Fig. 1. Silicon fan-shaped resonator with notched beam specimen. 
reactive etching with $\mathrm{SF}_{6}$ and $2 \mathrm{~s}$ of polymer deposition with $\mathrm{C}_{4} \mathrm{~F}_{8}$. The flows of $\mathrm{SF}_{6}$ and $\mathrm{C}_{4} \mathrm{~F}_{8}$ gases are 130 and $85 \mathrm{sccm}$, respectively. Third, after removing the photoresist on the top silicon layer, DRIE is performed with a sequence of $8 \mathrm{~s}$ of etching and $12 \mathrm{~s}$ of passivation from the backside to reach the $\mathrm{SiO}_{2}$ interlayer. During the backside etching, the silicon structure in the top silicon layer is protected by coating the photoresist. Finally, the photoresist and $\mathrm{SiO}_{2}$ interlayer were removed to release the movable part of the device. The beam and notch are aligned to $<110>$ crystallographic orientation. Because the rotational center of the movable part is at the notch tip of the specimen, the stress generated from a small electrostatic force is efficiently concentrated at the notch tip. This indicates that it is easy to identify the fracture point that is necessary for analyzing and understanding the fatigue fracture. In the device used in this study, the estimated value of the stress concentration factor using finite element method (FEM) analysis was 4.28.

\subsection{Experimental procedure}

The fatigue tests were conducted in an environmental chamber capable of controlling temperature and humidity. Figure 2 shows photographs of the custom-made measurement setup consisting of an environmental chamber, a heating system, a moisture generator unit, an electrical control system, and an observation system. The test temperature is controlled by the heater in the chamber. The air, supplied from compressed air, was passed through a bubbler placed inside
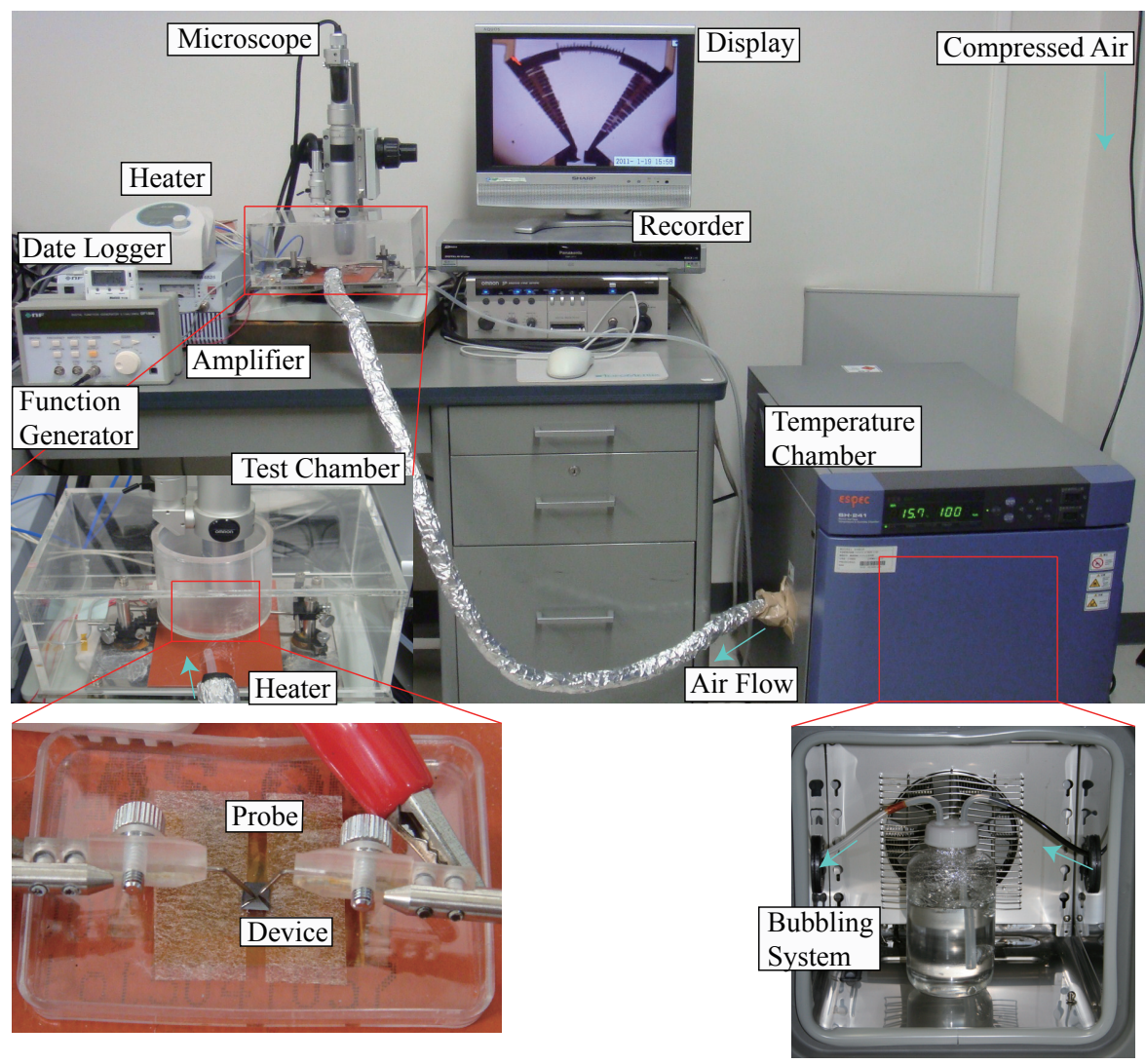

Fig. 2. (Color online) Experimental setup with environmental chamber. 
a thermohygrostat just before entering the chamber to maintain the temperature and humidity in the chamber. The temperature and humidity inside the chamber were monitored using a thermohygrometer and the data was logged during the test.

The test consisted of four series at two different temperatures and two different humidity conditions. Test conditions are shown in Table 1. We selected humidity because absolute humidity is similar at each temperature. Before starting the test, the testing device was placed inside the chamber and maintained for 60 min to stabilize the test condition.

In this test, $\mathrm{AC}$ voltage with $\mathrm{DC}$ bias that is half of the amplitude is applied as a driving signal. The frequency responses of the device were monitored using a microscope, and images obtained using a charge-coupled device (CCD) camera attached to the microscope were recorded on a hard disc. The captured images from recording data were used to detect the deflection angle under the applied voltage on both left and right sides by comparing the images before and during the test. Fatigue fracture point was also detected from recording data and then the number of cycles was calculated from the driving time of the device.

\section{Results and Discussion}

We characterized the fundamental frequency response of the device at first. The structure resonates approximately at the frequency of $49.4 \mathrm{kHz}$. Figure 3 shows the frequency responses at

Table 1

Environmental conditions in fatigue test.

\begin{tabular}{lclll}
\hline Temperature $\left({ }^{\circ} \mathrm{C}\right)$ & \multicolumn{2}{c}{25} & & \\
\hline Relative humidity $(\mathrm{RH})(\%)$ & 30 & 85 & 0.5 & 15 \\
Absolute humidity $\left(\mathrm{g} / \mathrm{m}^{3}\right)$ & 6.918 & 19.60 & 0.9861 & 29.58 \\
\hline
\end{tabular}

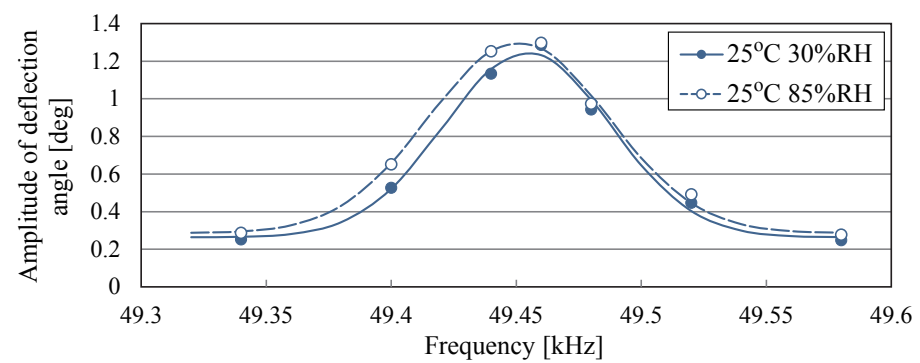

(a)

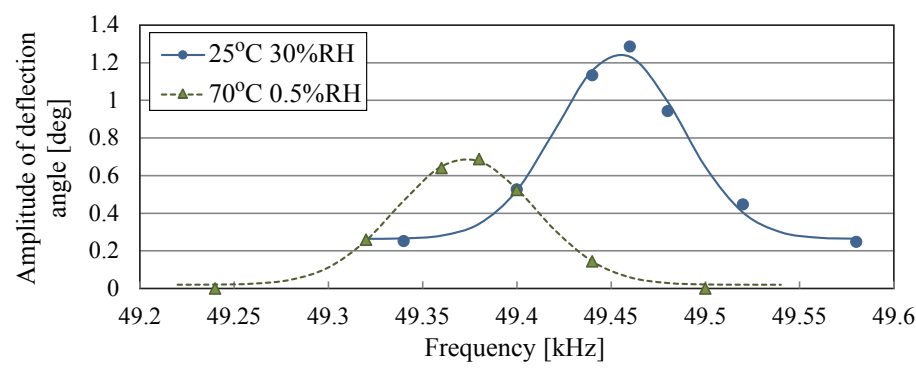

(b)

Fig. 3. (Color online) Frequency responses of microresonator in oscillation amplitude at applied voltage of $60 \mathrm{~V}$. 
different temperatures and humidity conditions. As the humidity changed at the same temperature, the resonant frequency and quality factor $(Q)$ were almost the same [Fig. 3(a)]. The damping effect due to surrounding moisture can be neglected in this work. However, the resonant frequency and the maximum rotation angle decreased as the temperature is increased [Fig. 3(b)]. The expression of Young's modulus and temperature in the case of single-crystal silicon is given as follows: ${ }^{(2)}$

$$
\frac{E_{\mathrm{Si}}(T)}{E_{\mathrm{Si}}\left(20{ }^{\circ} \mathrm{C}\right)}=1.019-9.385 \times 10^{-4} \mathrm{~T},
$$

where $E_{\mathrm{Si}}$ is the Young's modulus of silicon and $T$ is temperature. The rigidity of the structure decreases at high temperature. The degradation of rigidity resulted in the low resonant frequency. Moreover, thermoelastic dissipation (TED) affected the change in frequency responses due to temperature. ${ }^{(18)}$

Fatigue properties under different environmental conditions are shown in Fig. 4, comparing the effect of humidity. To evaluate the stress amplitude of the device, a FEM method was used to obtain the relationship between the maximum stress generated at the notch tip and the deflection angle of a fan-shaped movable part. In the FEM, the value of Young's modulus at $70{ }^{\circ} \mathrm{C}$ was $4 \%$ lower than that at $25^{\circ} \mathrm{C}$ according to Eq. (1). The stress amplitude calculated from both right and left deflection angles was measured by using captured images during the fatigue test. Fatigue life, which is the number of cycles until fracture, increases with decreasing amplitude of applied stress under all environmental conditions in the same way as in the previous studies. ${ }^{(8-14)}$ The slopes of S$\mathrm{N}$ curves were almost parallel under $25^{\circ} \mathrm{C}-30 \% \mathrm{RH}, 25^{\circ} \mathrm{C}-85 \% \mathrm{RH}$, and $70{ }^{\circ} \mathrm{C}-15 \% \mathrm{RH}$ conditions. At $25{ }^{\circ} \mathrm{C}$ [Fig. 4(a)], the S-N curve is higher at $30 \% \mathrm{RH}$ than at $85 \% \mathrm{RH}$. This result indicates that the fatigue strength of single-crystal silicon was greater under lower humidity condition. In the case of $25{ }^{\circ} \mathrm{C}$, the fatigue strength at $30 \% \mathrm{RH}$ is $5 \%$ higher than at $85 \% \mathrm{RH}$. However, the $\mathrm{S}-\mathrm{N}$ curve at $70{ }^{\circ} \mathrm{C}-15 \% \mathrm{RH}$ is $3.5 \%$ lower than at $25{ }^{\circ} \mathrm{C}-30 \% \mathrm{RH}$. This degradation of fatigue strength is related to the degradation of static strength at high temperature. The fracture strength of single-crystal silicon decreases with temperature higher than room temperature. ${ }^{(1,2)}$ At $70{ }^{\circ} \mathrm{C}$, the $\mathrm{S}-\mathrm{N}$ curve was less steep in slope at $0.5 \% \mathrm{RH}$ than at $1.5 \% \mathrm{RH}$ and intersected with the $\mathrm{S}-\mathrm{N}$ curve at $1.5 \% \mathrm{RH}$. It is noted that the fatigue life is more sensitive to the applied stress and the fatigue strength is relatively

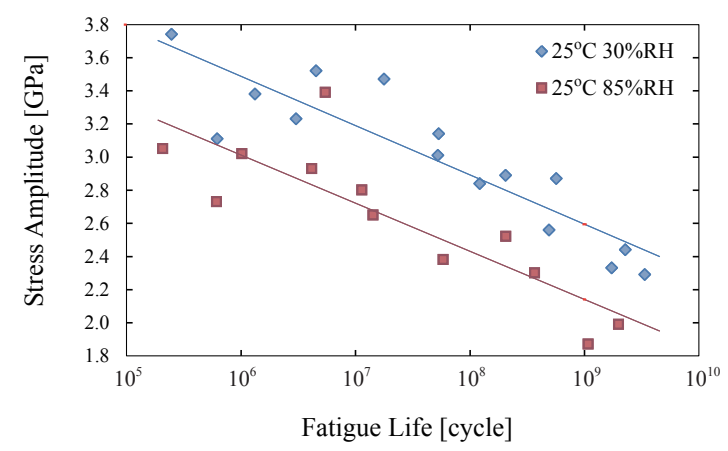

(a)

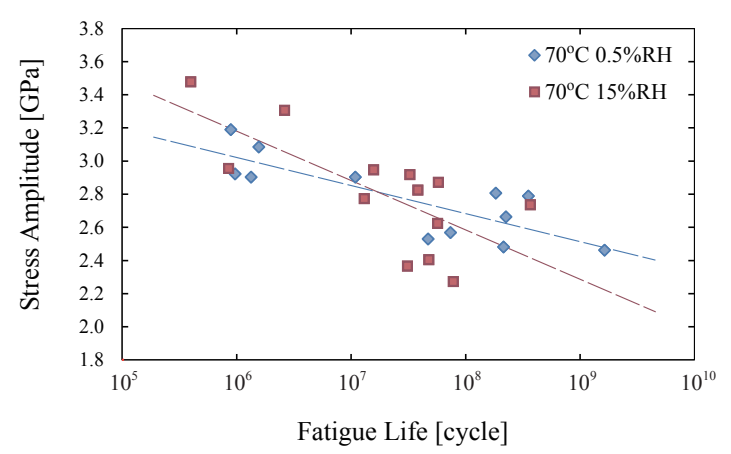

(b)

Fig. 4. (Color online) S-N plots of fatigue testing results in various environments at (a) 25 and (b) $70{ }^{\circ} \mathrm{C}$. 
high at high temperature under low-humidity condition. At a high cycle range over $10^{8}$ cycles, the fatigue strength increases with decreasing humidity at any temperature.

The oxidation of silicon, which involves $\mathrm{H}_{2} \mathrm{O}$ as an oxidizer, is much faster under wet condition than under dry condition, as is well known in the oxidation of a semiconductor. At room temperature, as the humidity increases, the thickness of a water-adsorbed layer on silicon increases, ${ }^{(19-21)}$ resulting in a thicker oxide layer at around room temperature. The thickness of the oxidation layer on the silicon surface measured by ellipsometry after a fatigue test at high humidity was 1.5-2 times higher than that measured at low humidity. The oxide layer was the possible cause of the degradation of the fatigue properties of single-crystal silicon, as another researcher reported.

The scanning electron microscopy (SEM) images of the failure specimen during the fatigue tests are shown in Fig. 5. The fracture specimens have a flat and smooth fracture surface of the (110) plane. However, there were three types of crack initiation from the notch tip. In the case shown in Fig. 5(a), the crack initiated from the notch tip that formed the smooth side line of the fracture surface. This type of fracture was observed at a small stress amplitude that occurred in

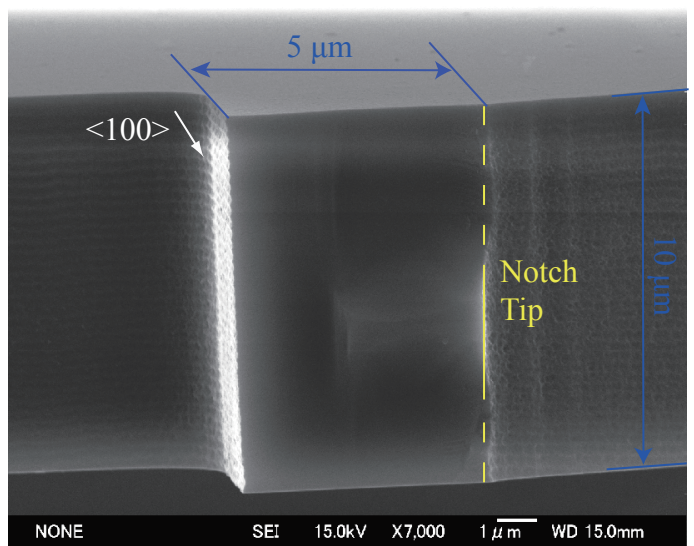

(a)

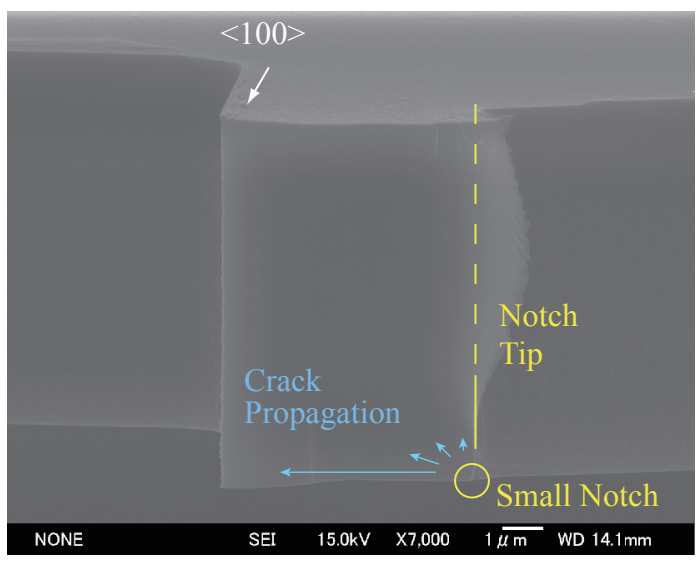

(c)
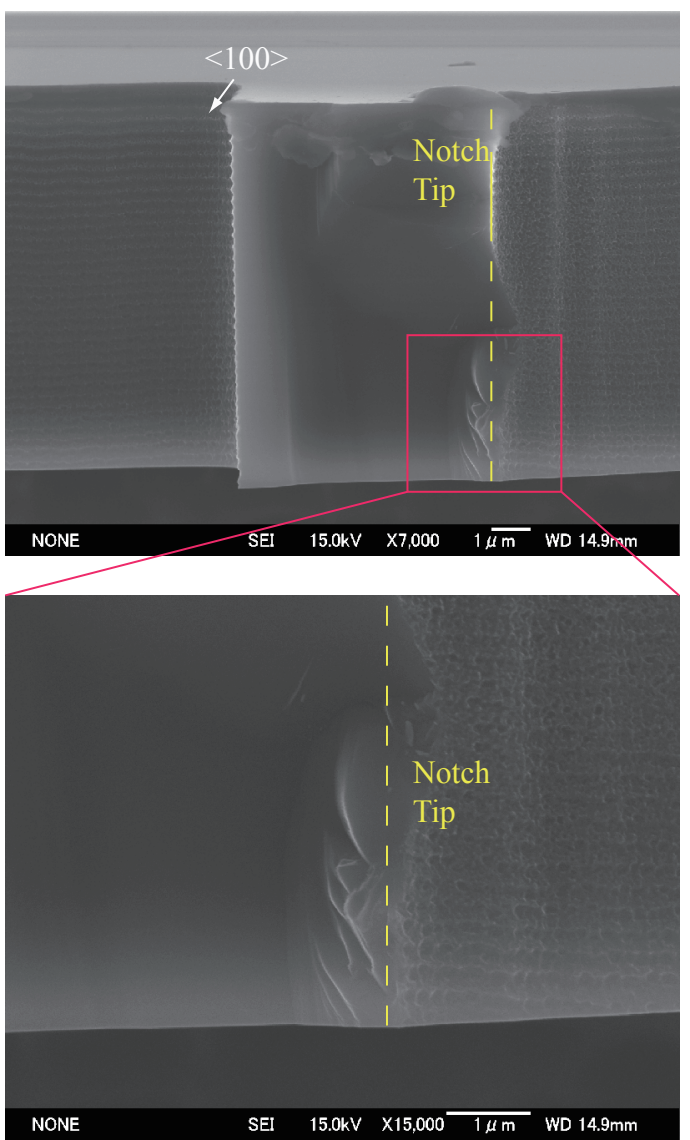

(b)

Fig. 5. (Color online) SEM images of fracture surface of microbeam fractured at a stress level of (a) low applied stress $\left(2.44 \mathrm{GHz}, 2.28 \times 10^{9}\right.$ cycles), (b) high applied stress $\left(3.07 \mathrm{GHz}, 6.99 \times 10^{6}\right.$ cycles), and (c) other conditions $\left(2.68 \mathrm{GHz}, 7.42 \times 10^{7}\right.$ cycles $)$. 
the cracks propagating along the $\langle 110>$ direction, which is a simple cleavage fracture. Figure $5(\mathrm{~b})$ shows that the notch tip on the fracture surface has straight-line-like striation in a particular part. The specimens with large stress amplitude and low fatigue cycle demonstrated this tendency. It was considered that the specimen fractured by cleavage; however, the internal energy produced from applied stress was consumed during the propagation of the main crack to create both fracture surfaces and other small cracks on and near the fracture surface. The last type of specimen fractured at the bottom of the notch tip and formed a curved surface as the crack propagated to the top of the specimen, as shown in Fig. 5(c). This fracture resulted from the morphology of the sidewall surface consisting of scalloping due to the Bosch process and a small notch at the bottom due to side etching. The overetching to an active layer of an SOI wafer caused this kind of bottom notch. In this case, the small notch caused by overetching on the notch tip is the origin of the fatigue fracture. The generated crack mainly propagated along the $\langle 110\rangle$ direction, that is, crack opening direction according to the applied stress. The cracks that proceeded to another direction formed a curved fracture surface on the top side. A different fracture mechanism was observed between the different applied stresses. However, no difference due to environmental conditions was observed. Moreover, it was almost impossible to identify the fracture initiating point, which is on the surface of the oxide layer or on the interface between the oxide layer and silicon.

\section{Conclusions}

Fatigue tests of single-crystal silicon using a MEMS resonator were performed at different temperatures and humidity conditions. The test humidity conditions were determined to be similar to the absolute humidity at each temperature.

The measurement results demonstrate that humidity has a large effect on fatigue strength and fatigue life, particularly at low temperature. However, no difference was observed at different temperatures, despite the change in the frequency response that was related to the change in Young's modulus and TED. The morphology of the fracture surface was only related to the applied maximum stress and of no relation to environmental conditions.

\section{References}

1 T. Namazu, Y. Isono, and T. Tanaka: J. Microelectromech. Syst. 11 (2002) 125.

2 S. Nakao, T. Ando, M. Shikida, and K. Sato: J. Micromech. Microeng. 16 (2006) 715.

3 X. Han, K. Zheng, Y.-F. Zhang, X. Zhang, Z. Zhang, and Z. L. Wang: Adv. Mater. 19 (2007) 2112.

4 C. St. John: Philos. Mag. 32 (1975) 1193.

5 S. Nakao, T. Ando, M. Shikida, and K. Sato: J. Micromech. Microeng. 18 (2008) 015026.

6 B. Kim, R. N. Candler, M. A. Hopcroft, M. Agarwal, W.-T. Park, and T. W. Kenny: Sens. Actuators, A 136 (2007) 125.

7 S. H. Choa: Microelectron. Reliab. 45 (2005) 361.

8 P. O. Theillet and O. N. Pierron: Appl. Phys. Lett. 94 (2009) 181915.

9 T. Tsuchiya, A. Inoue, J. Sakata, M. Hashimoto, A. Yokoyama, and M. Sugimoto: Tech. Dig. of the 16th Sensor Symposium (1998) p. 277.

10 T. Ando, M. Shikida, and K. Sato: Sens. Actuators, A 93 (2001) 70.

11 C. L. Muhlstein, S. B. Brown, and R. O. Ritchie: J. Microelectromech. Syst. 10 (2001) 593.

12 T. Ikehara and T. Tsuchiya: J. Micromech. Microeng. 18 (2008) 075004.

13 C. L. Muhlstein, E. A. Stach, and R. O. Ritchie: Acta Mater. 50 (2002) 3579.

14 H. Kahn, R. Ballarini, J. J. Bellante, and A. H. Heuer: Science 298 (2002) 1215.

15 S. Kamiya, Y. Ikeda, J. Gaspar, and O. Paul: Sens. Actuators, A 170 (2011) 187. 
16 D. H. Alsem, O. N. Pierron, E. A. Stach, C. L. Muhlstein, and R. O. Ritchie: Adv. Eng. Mater. 9 (2007) 15.

17 J. A. Connally and S. B. Brown: Science 256 (1992) 1537.

18 A. E. Duwel, R. N. Candler, T. W. Kenny, and M. Varghese: J. Microelectromech. Syst. 15 (2006) 1437.

19 T. Takahagi, H. Sakaue, and S. Shingubara: Jpn. J. Appl. Phys. 40 (2001) 6198.

20 D. B. Asay and S. H. Kim: J. Phys. Chem. B 109 (2005) 16760.

21 P. O. Theillet and O. N. Pierron: Sens. Actuators, A 171 (2011) 375. 